University of Nebraska - Lincoln

DigitalCommons@University of Nebraska - Lincoln

Faculty Publications in Computer \& Electronics Electrical \& Computer Engineering, Department Engineering (to 2015)

2006

\title{
Modeling Dual Polarization Wireless Fading Channels using Quaternions
}

\author{
B. J. Wysocki \\ University of Nebraska-Lincoln \\ Tadeusz Wysocki \\ University of Nebraska-Lincoln, wysocki@uow.edu.au \\ J. Seberry \\ University of Wollongong
}

Follow this and additional works at: https://digitalcommons.unl.edu/computerelectronicfacpub

Part of the Computer Engineering Commons

Wysocki, B. J.; Wysocki, Tadeusz; and Seberry, J., "Modeling Dual Polarization Wireless Fading Channels using Quaternions" (2006). Faculty Publications in Computer \& Electronics Engineering (to 2015). 27. https://digitalcommons.unl.edu/computerelectronicfacpub/27

This Article is brought to you for free and open access by the Electrical \& Computer Engineering, Department of at DigitalCommons@University of Nebraska - Lincoln. It has been accepted for inclusion in Faculty Publications in Computer \& Electronics Engineering (to 2015) by an authorized administrator of DigitalCommons@University of Nebraska - Lincoln. 


\title{
Modeling Dual Polarization Wireless Fading Channels using Quaternions
}

\author{
B.J. Wysocki, T.A. Wysocki, Senior Member IEEE, and J. Seberry, Senior Member IEEE \\ University of Wollongong, Australia \\ \{wysocki, bjw, jennie\}@uow.edu.au
}

\begin{abstract}
In the letter we describe signal transmitted between two dual-polarized antennas using a quaternion notation. The channel can then be modeled by a single quaternion gain, instead of a matrix of four complex gains as it is done in a classical approach. The model is very useful as it allows for a simple accommodation of any polarization twist between the transmit and receive antennas, as well as, modeling random fading in both polarizations with or without cross-polar scattering.
\end{abstract}

\section{Introduction}

Polarization diversity has been widely studied in the past, e.g. [1], and its diversity gain under Rayleigh fading considered in [2]. Recently, the demand for high rates in mobile communications has again raised the interest in applying polarization diversity, often together with other forms of diversity, like those considered in [3] or [4]. It has been shown there that polarization diversity can significantly add to the performance improvements offered by other diversity techniques, and be nearly as effective as spatial diversity for base station antennas, without a noticeable increase in their dimensions. In addition, the use of dual-polarized antennas for both transmitter and receiver combined with channel coding has been studied in [5].

The transmission channel for the case when dualpolarized antennas are used at both transmitter and receiver is usually described using four complex coefficients that leads to significant complexities when more than one antenna pair is considered. In this letter, we propose a simple mathematical model to describe such transmission channels using quaternions [6], where the channel is described by its gain coefficient being a single quaternion number.

The paper is organized as follows. In Section II, we introduce the description of the transmitted signal using quaternion numbers for dual-polarized antenna systems. The channel model is introduced in Section III. Simulation results are presented in Section IV, while Section V concludes the paper.

\section{Signal Description}

In [7], Isaeva and Sarytchev showed that the polarization state of an electromagnetic wave can be nicely modeled by means of quaternion representation, whereby two complex signal constellations

$$
z_{1}=x_{1}+i y_{1} \text { and } z_{2}=x_{2}+i y_{2}
$$

are orthogonal to each other on a polarization plane, just forming a quaternion number:

$$
s=z_{1}+z_{2} j=x_{1}+i y_{1}+j x_{2}+k y_{2}
$$

where $i, j$, and $k$ are hypercomplex numbers [7] satisfying

$$
i^{2}=j^{2}=k^{2}=-1 \text { and } i j k=-1
$$

In general, a quaternion number $a$ is defined as a linear combination of hypercomplex numbers $i, j$, and $k$, i.e.

$$
a=a_{1}+a_{2} i+a_{3} j+a_{4} k
$$

where $a_{m} ; m=1,2,3,4$, are real numbers. From (3), the following equalities can be drawn

$$
\begin{gathered}
i j=-j i=k \\
k i=-i k=j \\
j k=-k j=i
\end{gathered}
$$

which means that multiplication of quaternion numbers is not commutative. A product $a b$ of two quaternion numbers $a$ and $b$ is thus given by:

$$
\begin{aligned}
a b & =a_{1} b_{1}-a_{2} b_{2}-a_{3} b_{3}-a_{4} b_{4} \\
& +\left(a_{1} b_{2}+a_{2} b_{1}+a_{3} b_{4}-a_{4} b_{3}\right) i \\
& +\left(a_{1} b_{3}-a_{2} b_{4}+a_{3} b_{1}+a_{4} b_{2}\right) j \\
& +\left(a_{1} b_{4}+a_{2} b_{3}-a_{3} b_{2}+a_{4} b_{1}\right) k
\end{aligned}
$$

and, of course, $a b \neq b a$.

A rotation $s_{\varphi}$ of the signal $s$ on a polarization plane by an angle of $\varphi$ can be simply represented in the quaternion notation as:

$$
\begin{aligned}
s_{\varphi} & =s e^{j \varphi}=\left(z_{1}+z_{2} j\right) e^{j \varphi} \\
& =\left(z_{1}+z_{2} j\right)[\cos (\varphi)+j \sin (\varphi)]
\end{aligned}
$$

Similarly, any change to the polarization bases, e.g. due to differences between transmit and receive antenna alignments, can be easily represented in the quaternion notation, as long as the polarization bases are orthogonal [7]. 


\section{Channel Model}

Conventionally, transmission between two dualpolarized antennas (e.g. having both vertical and horizontal polarizations) is considered in a similar way as if there were 2 transmit and 2 receive antennas, resulting in 4 sub-channels, each characterized by its own complex gain (see Figure 1.a) much the same as in case of a 2-input 2-output MIMO system [8]. Assuming that an input signal to transmit antenna is of the form given by (1), with $z_{1}$ transmitted in the horizontal polarization and $z_{2}$ through the vertical one, the received signals $r_{1}$ and $r_{2}$ in the horizontal and vertical polarization of the receive antenna are:

$$
\begin{aligned}
& r_{1}=z_{1} h_{h h}+z_{2} h_{v h} \\
& r_{2}=z_{1} h_{h v}+z_{2} h_{v v}
\end{aligned}
$$

where $h_{h h}, h_{v h}, h_{h v}$, and $h_{v v}$ are complex channel gain coefficients for transmissions between horizontal and horizontal, vertical and horizontal, horizontal and vertical and vertical and vertical antennas, respectively.

(a)

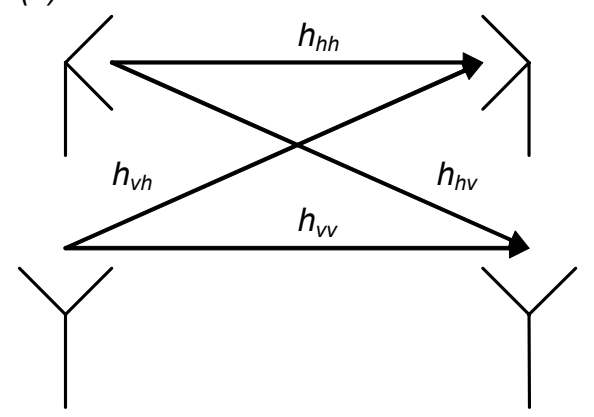

(b)

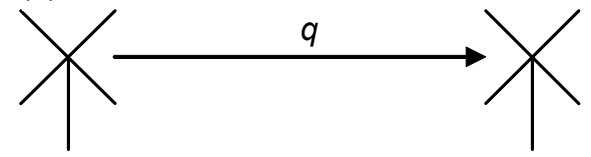

Figure 1: Schematic diagrams of channels utilizing dual-polarized antennas; (a) classical approach - the horizontally and vertically polarized antennas are considered separately and each of the channel gains, $h_{h h}$, $h_{v h}, h_{h v}$, and $h_{v v}$ are complex numbers, (b) proposed model - the channel between two dual-polarized antennas is described by a single quaternion gain $q$.

Hence, the output $r$ of the receive antenna system in the quaternion notation is

$$
r=r_{1}+r_{2} j
$$

We propose an alternative channel model, where the channel between the dual-polarized transmit and receive antennas is described by a single quaternion gain, $q=q_{1}+q_{2} i+q_{3} j+q_{4} k$, as illustrated in Figure 1.b. In this model, the received signal $\mathrm{r}$ is given by

$$
r=s q
$$

and estimation of the channel gain for performing ML decision involves estimation of just 4 real values that is half of what is required in the classical approach. From formulae (8) and (9), the relationship between the classical and the proposed models can be easily found, i.e.

$$
\begin{aligned}
\operatorname{Re}\left\{h_{h h}\right\} & =\operatorname{Re}\left\{h_{v v}\right\}=q_{1} \\
\operatorname{Im}\left\{h_{h h}\right\} & =-\operatorname{Im}\left\{h_{v v}\right\}=q_{2} \\
-\operatorname{Re}\left\{h_{v h}\right\} & =\operatorname{Re}\left\{h_{h v}\right\}=q_{3} \\
\operatorname{Im}\left\{h_{v h}\right\} & =\operatorname{Im}\left\{h_{h v}\right\}=q_{4}
\end{aligned}
$$

where $\operatorname{Re}\{\cdot\}$ and $\operatorname{Im}\{\{\}$ mean real and imaginary values, respectively.

The ML decision, when the proposed model is used, means finding a signal $\tilde{S}$ in a constellation $S$ that minimizes, over all possible values of $s \in S$, the following decision metric

$$
|r-s q|^{2}=(r-s q)(r-s q)^{Q}
$$

where $(\cdot)^{Q}$ denotes the quaternion transform. For a given quaternion number $a$, its quaternion transform $a^{Q}$ is

$$
a^{Q}=a_{1}-a_{2} i-a_{3} j-a_{4} k
$$

which, based on (3), (4), and (5), yields

$$
a a^{Q}=a^{Q} a=|a|^{2}=a_{1}^{2}+a_{2}^{2}+a_{3}^{2}+a_{4}^{2}
$$

The proposed model also simplifies simulation of transmission systems when dual-polarized antennas are employed. In the most general case of independent flat Rayleigh fading [9] for both polarizations and random cross-polar scattering, simulation involves just generation of four independent, zero-mean, Gaussian random variables, or in other words a random quaternion Gaussian variable. It is easy to notice, from equations (9) and (5) that at the receiver, each of the 4 signal amplitudes is then a zero-mean Gaussian random variable, which means that the envelopes of the received signal follow Rayleigh distribution for both polarizations. Some examples of channels, simulated using the proposed model, are presented in the next section.

\section{Simulation Results}

We used the developed channel model to simulate a Rayleigh fading environment for three different scenarios:

i) System utilizing a vertically polarized transmit antenna and a dual-polarized receive antenna with transmission involving both Rayleigh fading and random cross-polar scattering.

ii) System utilizing dual-polarized transmit and receive antennas but transmission involving just Rayleigh fading without any cross-polar scattering. 
iii) System utilizing dual-polarized transmit and receive antennas and transmission involving both random Rayleigh fading and random cross-polar scattering.

In all cases, we assumed a signal energy normalized to 1 and consisting of both real and imaginary components. The channel coefficient was also normalized to have no effect on the received channel energy, i.e. the sum of variances of $q_{1}, q_{2}, q_{3}$, and $q_{4}$ being equal to 1 . For cases (i) and (iii) that meant all $q_{1}, q_{2}, q_{3}$, and $q_{4}$ being i.i.d. Gaussian random variables with zero mean and variances equal to 0.25 , while for case (i) $q_{3}=q_{4}=0$, and $q_{1}$, and $q_{2}$ being i.i.d. Gaussian random variables with zero mean and variances equal to 0.5. The simulation was repeated 1000 times, and the resulting variations in the received signal envelope magnitudes for both polarizations are plotted in Figures 2.a., 3.a., and 4.a. for a horizontal polarization and Figure 2.b., 3.b.,
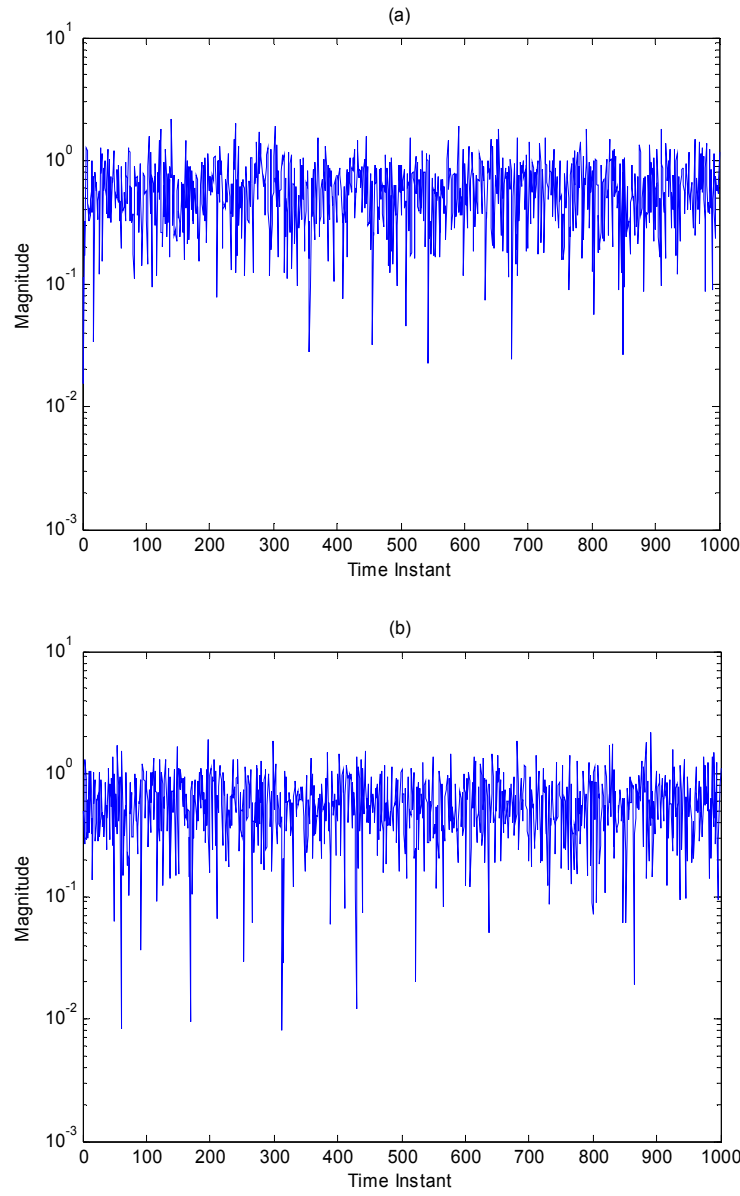

Figure 2: Plots of the magnitudes of signal envelopes for scenario (i); (a) horizontal polarization, (b) vertical polarization.
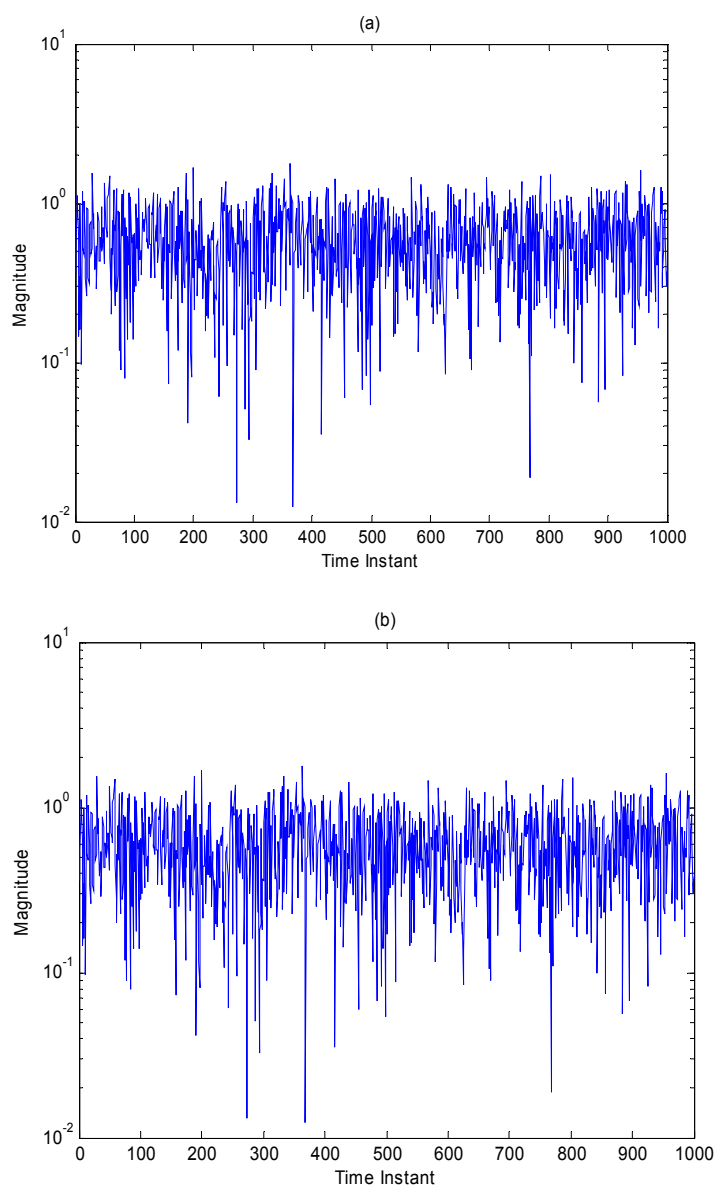

Figure 3: Plots of the magnitudes of signal envelopes for scenario (ii); (a) horizontal polarization, (b) vertical polarization.

and 4.b., for the vertical polarization for scenarios (i), (ii), and (iii), respectively. It is interesting to notice that the correlation coefficients between the values of envelope magnitudes for the horizontal and vertical polarizations for scenarios (i), (ii), and (iii) are 0.0144, 1.0 , and 0.0095 , respectively.

\section{Conclusions}

In the paper we proposed a simple mathematical model for wireless fading channels where dual-polarized antennas are used. The model is based on describing the dual-polarized signal by means of quaternion representation, which leads to a channel described by its gain being a single quaternion variable. The model is very useful as it allows for a simple accommodation of any polarization twist between the transmit and receive antennas, as well as, modeling random fading in both polarizations with or without cross-polar scattering. 

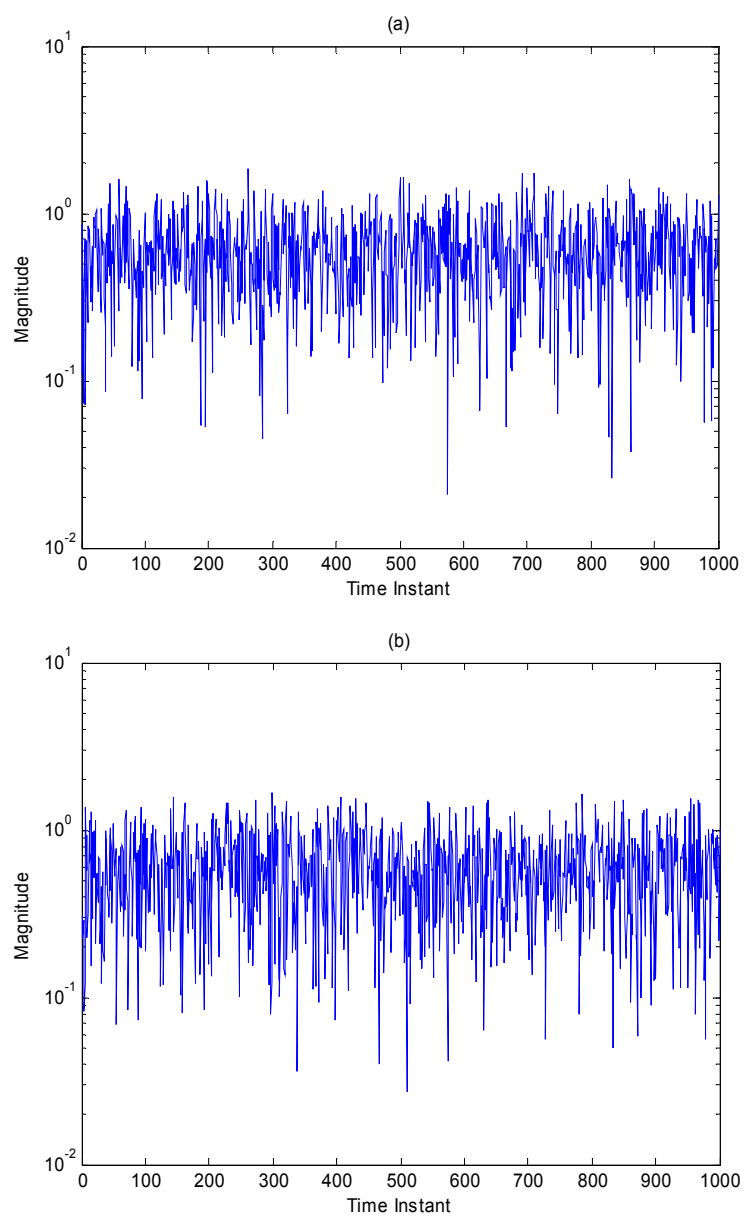

Figure 4: Plots of the magnitudes of signal envelopes for scenario (iii); (a) horizontal polarization, (b) vertical polarization.

\section{References}

[1] B.S.Collins, "Polarization-diversity antennas for compact base stations," Microwave Journal, Vol. 43, No 1, pp 76 - 88, January 2000.

[2] B.S.Collins, "The effect of imperfect antenna cross-polar performance on the diversity gain of a polarization-diversity system," Microwave Journal, Vol. 43, No4, pp. 84 - 94, April 2000.

[3] C.B.Dietrich, K.Dietze, J.R.Nealy, and W.L.Stutzman, "Spatial, polarization, and pattern diversity for wireless handheld terminals," IEEE Trans. Antennas Propagat., vol. 49 , pp. $1271-1281,2001$.

[4] R.U.Nabar, H.Bölcskei, V.Erceg, D.Gesbert, and A.J.Paulraj, "Performance of multiantenna signalling techniques in the presence of polarization diversity," IEEE Trans. Signal Processing, vol. 50, pp. 2553 - 2562, 2002.

[5] Z.Zhao, S.stapleton, and J.K.Cavers, "Analysis of polarization diversity scheme with channel codes," Proc. IEEE VTC'99, pp. 1377 - 1381, 1999.
[6] Altmann, S. L. Rotations, Quaternions, and Double Groups. Oxford, England: Clarendon Press, 1986.

[7] O.M.Isaeva, and V.A.Sarytchev: "Quaternion presentations polarization state," Proc. $2^{\text {nd }}$ IEEE Topical Symposium of Combined OpticalMicrowave Earth and Atmosphere Sensing, Atlanta, GA USA, 3-6 April 1995, pp.195-196.

[8] S.Haykin, and M.Moher, Modern Wireless Communications, Pearson Prentice Hall, Upper Saddle River, 2005. 